\title{
Exploring Autistic Adults' Psychosocial Experiences Affecting Beginnings, Continuity, and Change in Camouflaging Over Time: A Qualitative Study
}

\begin{abstract}
Authors and Affiliations
Beatrice Rui Yi LOO, Department of Psychology, National University of Singapore (Joint First Author) Truman Jing Yang TEO, Department of Psychology, National University of Singapore (Joint First Author) Melanie JiaMin LIANG, Department of Psychology, National University of Singapore Dawn-Joy LEONG, Autistic Consultant, Independent Researcher and Multi-Artist, Singapore.

Diana Weiting TAN, School of Education, Macquarie University, Australia; School of Psychological Science, University of Western Australia, Australia; Telethon Kids Institute, Australia

Sici ZHUANG, School of Psychological Science, University of Western Australia

Laura HULL, Department of Clinical, Educational and Health Psychology, University College London

Lucy A. LIVINGSTON, School of Psychology, Cardiff University and Social, Genetic \& Developmental Psychiatry Centre, Institute of Psychiatry, Psychology and Neuroscience, King's College London Will MANDY, Department of Clinical, Educational and Health Psychology, University College London Francesca HAPPÉ, Social, Genetic \& Developmental Psychiatry Centre, Institute of Psychiatry, Psychology and Neuroscience, King's College London

Iliana MAGIATI, Department of Psychology, National University of Singapore; Dr. Magiati is now at the School of Psychological Science, University of Western Australia. (Senior Author)
\end{abstract}




\begin{abstract}
Many autistic people develop camouflaging strategies to mask or compensate for their underlying autism-related differences in order to get by in predominantly non-autistic societies. Autistic adults have described camouflaging as a "lifetime of conditioning to act normal", suggesting that camouflaging develops over the autistic person's lifespan. Yet, very little is so far known about the course of camouflaging motivations and behaviours over time or the psychosocial factors that may influence autistic people's camouflaging trajectories. In an exploratory qualitative study within an Asian sociocultural context, we interviewed 11 Singaporean autistic adults in depth about their camouflaging experiences throughout their lives. We aimed to better understand the beginnings, continuity and changes in their camouflaging motivations and strategies over time, and related psychosocial influences. Interviews were transcribed verbatim and thematically analysed. Twenty-six themes were identified, and were organised across four phases - pre-camouflaging, beginnings, continuity, and change over time. The earliest camouflaging motivations were predominantly relational, linked to a negative self-identity shaped by adverse social experiences. Strategies were increasingly complex and better integrated into one's sense of self over time, or more selectively engaged in. Our findings highlight the role of psychosocial pressures precipitating camouflaging. They also emphasise the need for both individual psychological and societallevel changes to move from pathologizing autistic differences to acceptance, understanding, and inclusion, so as to reduce psychosocial pressures on autistic people of all ages to camouflage.
\end{abstract}




\section{Lay Abstract}

Over their lifetimes, many autistic people learn to hide their autism-related differences in order to forge and maintain relationships, find work and live independently in largely nonautistic societies. This masking of autistic differences is otherwise known as camouflaging. Autistic adults have described camouflaging to be a "lifetime of conditioning, [training] to act normal", involving "years of effort and practice". Yet little is known about why and how autistic people first start to camouflage, or why and how their camouflaging behaviours continue or change over time. We interviewed 11 Singaporean autistic adults who shared their camouflaging experiences throughout their lives. We found that autistic adults' earliest motivations to camouflage were largely related to the desire or need to connect with other people. They were also motivated by wanting to reduce or avoid difficult social experiences (such as being teased, bullied, or socially isolated), and linked to the adults' developing negative beliefs about themselves. Autistic adults shared that their camouflaging behaviours became more complex, and some actually became a part of their self-identity over time. Autistic adults also learnt to be more selective with camouflaging as they grew older, for example hiding their autism only in some contexts but not others. Our findings suggest that people should not pathologise or stigmatise autistic differences, as this may put pressure on autistic individuals to camouflage to fit into a largely non-autistic society. There is a need to move toward acceptance, understanding, and inclusion, to reduce the pressure on autistic people to hide their autism and many aspects of who they truly are. 


\section{Introduction}

Autism is a lifelong neurodevelopmental condition characterised by difficulties, differences, and strengths in social communication and interaction, intense and highly-focused interests and behaviours, and atypical sensory reactivity and preferences (APA, 2013). It is highly heterogeneous in terms of its behavioural and cognitive presentations, the extent of cooccurring medical and mental health conditions, levels of intellectual functioning and independence, and outcomes in adulthood (Howlin \& Magiati, 2017; Kuo et al., 2018; Lord et al., 2015; Lord et al., 2020). Autistic people are on average significantly more likely than nonautistic people to experience social vulnerability and isolation, including bullying, discrimination, and ostracism (Griffiths et al., 2019; Schroeder et al., 2014). Consequently, autistic people often report poorer quality of life, well-being, and independence than their nonautistic counterparts (see Magiati et al., 2014).

Several recent studies have suggested that some autistic people learn to cope with these challenging social experiences by developing strategies to "mask" or "camouflage" their autism. Camouflaging in autism has been defined as the use of strategies by autistic individuals to minimise the visibility of their autistic traits and associated differences or difficulties (Hull et al., 2017). Recent research has identified three broad types of camouflaging strategies (Hull et al., 2017; Livingston et al., 2019; 2020). Masking involves hiding one’s autism-related characteristics (e.g., minimising self-soothing behaviours). Although masking strategies do not necessarily support active two-way social participation, they enable autistic people to blend in and hide their autism (Hull et al., 2017; Livingston et al., 2019; 2020). Compensation is more cognitively demanding than other strategies as it involves learning and generating new social behaviours to fill the social and communication "gaps" between autistic and non-autistic people (Hull et al., 2017; Livingston et al., 2019). Compensation may be shallow, for instance laughing when others laugh after a joke without necessarily understanding why the joke was funny; or 'deeper', such as applying learnt social rules to interpret why a joke was funny, although the 
person camouflaging might not have intuitively understood this (Livingston \& Happe, 2017). Finally, assimilation involves making attempts to avoid or manage the discomfort an autistic person may often feel in social situations, without letting others notice that discomfort (Hull et al., 2018).

Broadly, autistic people camouflage to adapt to their predominantly non-autistic social environments for two main reasons: for interpersonal reasons (e.g., to form social relationships, increase social reputation, and/or reduce negative social experiences, such as bullying, social rejection, or stigma; see Bargiela et al., 2016; Cage \& Troxell-Whitman, 2019; Livingston et al., 2019; Pearson \& Rose, 2021); and/or for conventional purposes (e.g., getting a project done at work or school, or maintaining employment; see Cage \& Troxell-Whitman, 2019; Hull et al., 2017; Livingston \& Happé, 2017). These motivations highlight that many autistic people are acutely aware of the social demands in non-autistic social environments, and often feel pressured to camouflage to meet these social demands (Milton, 2012; Sasson et al., 2017).

Despite camouflaging bringing several potential benefits to autistic people, such as enabling them to form relationships and find employment (e.g., Cage \& Troxell-Whitman, 2019; Livingston et al., 2019), it has also consistently been described as stressful (Cage \& Troxell-Whitman, 2019), physically and mentally exhausting (Bradley et al., 2021; Cook, Crane, Bourne, et al., 2021; Hull et al., 2017), confusing and detrimental to one's self-identity (Leedham et al., 2019), and a potential barrier to timely and accurate autism diagnosis (Bargiela et al., 2016; Begeer et al., 2013) and appropriate support (e.g., in the workplace; Livingston et al., 2019). Cross-sectional self-report questionnaire studies to date have found that increased camouflaging behaviours are associated with a range of poorer mental health outcomes in autistic people, including depression, anxiety, and suicidality (Beck et al., 2020; Bernardin, Lewis, et al., 2021; Cassidy et al., 2018, 2020; Hull, Levy, et al., 2021). Autistic people have also often pinpointed camouflaging efforts as a major contributor to autistic burnout, characterised by chronic exhaustion, loss of skills, intensifying of one's autism-related traits, 
reduced tolerance to stimuli, and overall reduced functioning (Higgins et al., 2021; Raymaker et al., 2020).

\section{Continuity and Change in Camouflaging Across the Lifespan}

The Diagnostic and Statistical Manual of Mental Disorders-5 (DSM-5; APA, 2013) describes autism as a lifelong condition present since the early developmental period and recognises that autistic presentations may be hidden by strategies acquired by the autistic individual over time to cope with the social, communication, and sensory challenges of nonautistic environments (APA, 2013). While most camouflaging research to date has been with autistic adults, there is increasing qualitative (Bernardin, Mason, et al., 2021; Halsall et al., 2021; Cook et al., 2018; Tierney et al., 2016) and quantitative (Bernardin, Lewis, et al., 2021; Corbett et al., 2021; Hull, Petrides, \& Mandy, 2021) evidence that autistic children and adolescents, as young as seven years old (Dean et al., 2016), also engage in camouflaging. Masking autistic features during childhood and adolescence has also been suggested as a possible reason why some autistic individuals, especially women, are only diagnosed later in life (Gould, 2017). Autistic people have described camouflaging as a "lifetime of conditioning, [training] to act normal" (Cage \& Troxell-Whitman, 2019), involving “years of effort" and practice (Leedham et al., 2019), suggesting that camouflaging develops over the autistic person's lifespan, likely beginning in childhood.

With regards to camouflaging motivations, some autistic adults have reported that social motivations were more common in childhood and adolescence than in adulthood (Hull et al., 2017). Indeed, the most common reasons for camouflaging reported by autistic adolescents in a large survey study were to gain social acceptance and to avoid negative social experiences or perceptions (Bernardin, Lewis, et al., 2021). In contrast, camouflaging for conventional reasons, in both educational and employment settings, was more commonly described to occur in adulthood than in childhood (Bargiela et al., 2016; Cage \& Troxell-Whitman, 2019; Hull et al., 
2017), suggesting possible changes to social experiences, identity, or motivations for camouflaging over time.

Little is known about continuity and change in camouflaging strategies over time. Latediagnosed autistic women have recounted initially being passive and compliant in their primary school years as a way to blend in and hide their differences (Bargiela et al., 2016). Additionally, an observational study found that school-aged autistic girls often play in close proximity to their peers but not always in active engagement with them (Dean et al., 2016), suggesting that some autistic girls (and potentially boys) may engage in early camouflaging strategies that involve hiding one's autistic characteristics. In autistic adults, a variety of camouflaging strategies have been described, including masking, shallow and deep compensation, and assimilation (Hull et al, 2018; Livingston et al., 2020). In a novel study using Interpersonal Process Recall methodology, Cook, Crane, Hull, and colleagues (2021) identified 38 camouflaging behaviours used by autistic adults in daily interactions, some of which overlapped with those identified in previous camouflaging research, while others were newly reported, such as the use of deferential engagement (e.g., apologising or offering excuses for social missteps or poor social performance), minimising social risks, and reciprocal social behaviours. Yet, very little is known about what drives the emergence and development of different camouflaging behaviours over time, as to the best of our knowledge, no study to date has systematically explored autistic people's camouflaging beginnings and trajectories. In qualitative studies to date, autistic adults have not been invited specifically to recall and reflect on their earliest motivations to camouflage or how these motivations have evolved in their lives over time, though one study revealed qualitative themes related to how camouflaging strategies may have changed over time (Livingston, Shah, and Happé, 2019). Furthermore, the psychosocial circumstances or experiences that may have influenced the emergence and trajectory of camouflaging behaviours in autistic people have yet to be systematically explored.

\section{The Present Study}


In the present study, we explored the emergence of and developmental trajectories in camouflaging motivations and strategies over time through in-depth qualitative interviews. Psychological processes and social circumstances and experiences may differ - some factors may remain central to an autistic person's camouflaging experience over time, while the influence of others may change across different periods of their life (Bronfenbrenner, 1994; Lehman et al., 2017). Drawing from a dynamic biopsychosocial framework (Lehman et al., 2017), we explored intra-individual and sociocultural influences on the beginnings and development of autistic camouflaging over time. Exploring camouflaging developmentally and within a psychosocial framework may help to identify (i) early risk or protective psychological, developmental, and/or social experiences and (ii) specific periods or social contexts in which autistic people are more likely to experience pressures to camouflage. Such knowledge and understanding could in turn help reduce the pressure on autistic people to camouflage, and/or alleviate the costs to their mental wellbeing when engaging in camouflaging behaviours.

Thus, the present qualitative study aimed to investigate the following research questions:

(1) What are autistic adults' earliest recollections of the emergence of their camouflaging behaviours? And what individual psychological and sociocultural experiences/ circumstances motivated them to start camouflaging?

(2) In what ways do autistic adults' camouflaging behaviours and motivations remain stable or develop and change over time? And more specifically, what individual characteristics or social contexts/ psychological experiences influenced continuity and change in their camouflaging motivations and strategies over time?

Further, whilst the present study is not a direct investigation of the cultural effects on camouflaging, we explored these questions by interviewing Singaporean autistic adults within an Asian sociocultural context, whereas most, if not all, studies to date have been conducted in predominantly Western countries (mostly in the UK, US, and Australia). Thus, the themes and 
subthemes derived may also provide some insights into the potential influences of one's broader socio-cultural environment on camouflaging motivations.

\section{Method}

\section{Participants}

Participants (i) were at least 21 years old ${ }^{1}$, (ii) reported having received a formal autism diagnosis by a qualified professional in childhood, adolescence, or adulthood, and (iii) reported having engaged in or currently engaging in camouflaging behaviours on a brief screening question before the interview. Individuals who did not have adequate capacity to provide informed consent, could not verbally describe their experiences in English, or reported experiencing acute mental health difficulties currently or within the last six months ${ }^{2}$ were not included in the study.

Participants were recruited via personal and professional contacts of the study investigators, social media, support groups for autistic adults in Singapore, and by contacting autistic participants from a previous study by the last author who had given consent to be contacted about future studies. Interested participants completed a brief pre-screening questionnaire to ensure they met the above-mentioned inclusion criteria. Eleven autistic adults participated in this study, and their demographic characteristics are summarised in Table 1.

\footnotetext{
${ }^{1}$ The age when a person is legally considered an adult in Singapore, where this study took place, by common law is 21 years old.

${ }^{2}$ Individuals who responded "yes" to a screening question asking if they had experienced significant stress, severe emotional distress, abuse in a current relationship, or thoughts of harming themselves in the last six months were excluded from the study. This was because the student interviewers (first and second authors) were not trained or qualified to manage possible high-risk information being shared. Only two autistic adults who expressed interest in the study were excluded for this reason, and appropriate contacts for services and supports were provided to them.
} 
Table 1

Participants' Characteristics $(N=11)$

\begin{tabular}{|c|c|}
\hline & $\mathrm{N}$ or Mean $(\mathrm{SD})$ [Range] \\
\hline $\begin{array}{l}\text { Gender } \\
\qquad \begin{array}{l}\text { Male } \\
\text { Female }\end{array}\end{array}$ & $\begin{array}{l}9 \\
2\end{array}$ \\
\hline $\begin{array}{l}\text { Ethnicity }^{\mathrm{a}} \\
\text { Chinese } \\
\text { Malay }\end{array}$ & $\begin{array}{c}10 \\
1\end{array}$ \\
\hline Age (years) & $30.3(7.2)[22-45]$ \\
\hline Age at diagnosis (years) & $18.5(11)$ [3-44] \\
\hline $\begin{array}{l}\text { Autism diagnosis } \\
\text { Autism } \\
\text { Asperger's Syndrome } \\
\text { Autism Spectrum Disorder and } \\
\text { Asperger's Syndrome } \\
\text { b }\end{array}$ & $\begin{array}{l}3 \\
6 \\
2\end{array}$ \\
\hline $\begin{array}{l}\text { Highest education level attained } \\
\text { Apprenticeship/College/Diploma } \\
\text { Undergraduate degree } \\
\text { Postgraduate degree }\end{array}$ & $\begin{array}{l}5 \\
5 \\
1\end{array}$ \\
\hline $\begin{array}{l}\text { Employment status } \\
\text { Student } \\
\text { Unemployed } \\
\text { Full-time employment }\end{array}$ & $\begin{array}{l}4 \\
1 \\
6\end{array}$ \\
\hline $\begin{array}{l}\text { Autistic traits } \\
\text { AQ-Short Total Score }{ }^{c} \text { (min-max } \\
\text { score range 28-112) }\end{array}$ & 80.3 (11.2) [69-97] \\
\hline $\begin{array}{l}\text { Camouflaging Behaviours } \\
\text { CAT-Q Total Score (min-max score } \\
\text { range } 25-175 \text { ) }\end{array}$ & 102.9 (11.2) [81-116] \\
\hline
\end{tabular}

${ }^{a}$ Singapore is a multi-ethnic society and citizens comprise four main racial groups: Chinese (76\%), Malay (15\%), Indian (7\%), and Others (1.5\%; https://www.strategygroup.gov.sg/files/media-centre/publications/population-in-brief-2020.pdf).

\footnotetext{
${ }^{b}$ Participants were allowed to select as many diagnoses as applied to them. Two participants indicated a diagnosis of Autism Spectrum Disorder in addition to their diagnosis of Asperger's Syndrome.
}

${ }^{\mathrm{c} A Q}$-Short = Autism-Spectrum Quotient (Short Form; Hoekstra et al., 2011); CAT-Q = Camouflaging of Autistic Traits Questionnaire (Hull et al., 2018). 


\section{Materials and Measures}

\section{Demographics, Autistic Traits, and Camouflaging Measures}

Participants provided demographic information about themselves (see Table 1).

Then, they completed the 28-item Autism-Spectrum Quotient Short (AQ-Short;

Hoekstra et al., 2011; total score range 28-112; higher scores indicate greater autistic traits; see Table 1 for participants' scores). A score of more than 65 on the AQ-Short has a sensitivity of .97 and a specificity of .82 (Hoekstra et al., 2011) in differentiating autistic from non-autistic people; all participants' self-reported ratings were $>65$.

Finally, participants completed the Camouflaging Autistic Traits Questionnaire (CATQ; Hull et al., 2018) to confirm that participants have engaged in at least some camouflaging behaviours. The CAT-Q comprises 25 items and has a total and three-factor subscale scores Compensation, Masking, and Assimilation. Each item is rated on a 7-point Likert scale from 1 (strongly disagree) to 7 (strongly agree; total score range 25-175; higher scores indicate more camouflaging). The CAT-Q was initially validated in a sample of autistic $(\mathrm{n}=306)$ and nonautistic adults $(n=400)$ with the full scale demonstrating excellent internal consistency $(\alpha=$ $.94)$ and good test-retest reliability in an autistic subsample $(n=30)$ over three months (Pearson's $r=.77$; Hull et al., 2018).

\section{Interview Guide}

To develop the interview guide, we first extensively reviewed the camouflaging qualitative research published to date to identify gaps in the existing literature and pinpoint pertinent aspects of an autistic individual's camouflaging experiences to be further explored in depth with regards to the beginnings, continuity, and change in autistic camouflaging over time. Next, the first, second, and last authors, in consultation with and with guidance from our autistic 
consultant (fourth author, XX), developed a draft interview guide which they discussed and refined several times before obtaining inputs from and finalising with the other authors.

The interview guide was organised into two parts. In Part 1, participants' earliest recollections of camouflaging motivations and strategies were explored; in Part 2, participants were invited to recollect and reflect on their trajectories of camouflaging over time, specifically how their camouflaging has remained the same or changed over time and across different social contexts during their childhood, adolescence, and adulthood. Each of the two interview parts contained an opening question that was broad and open-ended, followed by prompting questions to facilitate greater detail of experiences and perspectives, used flexibly if and as needed (see Appendix 1 for the opening questions and sample follow-up questions within each main interview segment).

\section{Procedure}

This study was granted ethical approval by the [name of university withheld for blind peer review] (Ref. Number S-19-363).

Three days before the interview, participants were emailed a link to the main interview questions so that they could prepare if they wished to. We also asked if they required any accommodations that we could reasonably provide during the interview (e.g., interview room lighting, map directions, visual printouts of the questions). On the day of the interview, participants gave informed consent and completed the self-report measures before being interviewed.

Interviews were conducted in a quiet and private room at a training psychology clinic by the first and second authors guided and supervised by the senior author. As camouflaging is a highly personal experience and the interview potentially touched on sensitive or distressing past/ current social experiences, breaks were offered whenever needed. Interviews lasted no longer than 90 minutes (range 60-90 minutes), and all were recorded and transcribed verbatim. 
Finally, participants were debriefed and provided with an information sheet detailing mental health resources and local services from which they could seek support if needed. Participants received \$30 Singapore dollars for their participation via electronic transfer or in grocery vouchers and were additionally reimbursed up to \$20 Singapore dollars for transportation costs.

\section{Thematic Analysis}

All 11 interviews were transcribed verbatim, and the data were then coded and interpreted following the six phases of reflexive thematic analysis by Braun and Clarke (2006; see also Braun \& Clarke, 2019). As there are currently no specific empirical data or theories that describe the developmental trajectories of camouflaging in autistic individuals, transcripts were analysed through an inductive process, where codes and themes were generated and derived from descriptions in the data, rather than grounded in any pre-established theoretical framework. Themes were identified semantically and explicitly, but some a priori organisation was applied in the way the themes were structured into the two main domains of the study's research questions (Motivations/ Pressures; Strategies) and across time/ trajectory (precamouflaging, beginnings, continuity, and change). Some a priori terminology was also used (i.e., the masking, compensation, and assimilation terms/ constructs identified by Hull et al, 2018; Livingston et al., 2019) when the identified data themes were consistent with these terms.

In terms of the researchers' positionality, $\mathrm{XX}$ is an autistic researcher and artist and her lived experience of being autistic may have influenced her positionality. All researchers have received professional training in psychology. Some of the authors have lived or are living in Singapore and thus their positionality may have been influenced by their experiences of its sociocultural context $(\mathrm{XX}, \mathrm{XX}, \mathrm{XX}, \mathrm{XX}, \mathrm{XX}, \mathrm{XX})$. The authors have consulted with the autistic consultant, $\mathrm{XX}$, on the process and goals of the current study and view autism through a social 
model of disability, which could steer us towards identifying themes that consider psychosocial influences on and consequences of autistic individuals' camouflaging behaviour and trajectories.

In the first cycle of coding, and guided by the study's broad research questions, the first five interview transcripts were initially independently coded by XX and XX. Following this, data extracts and corresponding initial codes were discussed extensively between $\mathrm{XX}$ and $\mathrm{XX}$ and then reviewed and discussed in detail with $\mathrm{XX}$ until consensus was reached. After further consultation with $\mathrm{XX}$, the coding scheme's themes were provisionally finalised. These were then applied to the remaining transcripts in the second cycle of coding, again independently coded by the two first authors and discussed extensively with the last author until consensus was reached. Any further themes derived from the remaining manuscripts were integrated into the coding scheme, which was revised as necessary to ensure that the coding represented the primary content of all interviews comprehensively and parsimoniously. Before finalising the coding of the interviews, another consultation took place with XX to ensure relevance, accuracy, and clarity.

\section{Community Involvement}

The interview guide was developed in consultation with the fourth author (XX), an autistic researcher, advocate, and multi-artist, who shared her own experiences and perspectives on her camouflaging trajectory, as well as her understanding of other autistic adults' shared experiences, and who provided input and guidance on the ethical appropriateness, relevance, and clarity of the interview guide and questions for autistic adults. As summarised in the section above, XX also provided her input and thoughts on the themes derived from our thematic analysis which guided the revisions and final decisions with regards to the coding scheme.

\section{Results}


The interview guide was organised into questions focusing on motivations and questions on strategies, and so thematic analysis was carried out within each of these two domains.

\section{Themes}

Figure 1 visually summarises the domains (motivations, strategies) and the themes derived from the thematic analysis along the four developmental stages (pre-camouflaging, beginnings of camouflaging, continuity of camouflaging over time, and change in camouflaging over time). 


\section{Figure 1}

Overall Thematic Map of all Themes Relating to Camouflaging Motivations and Strategies

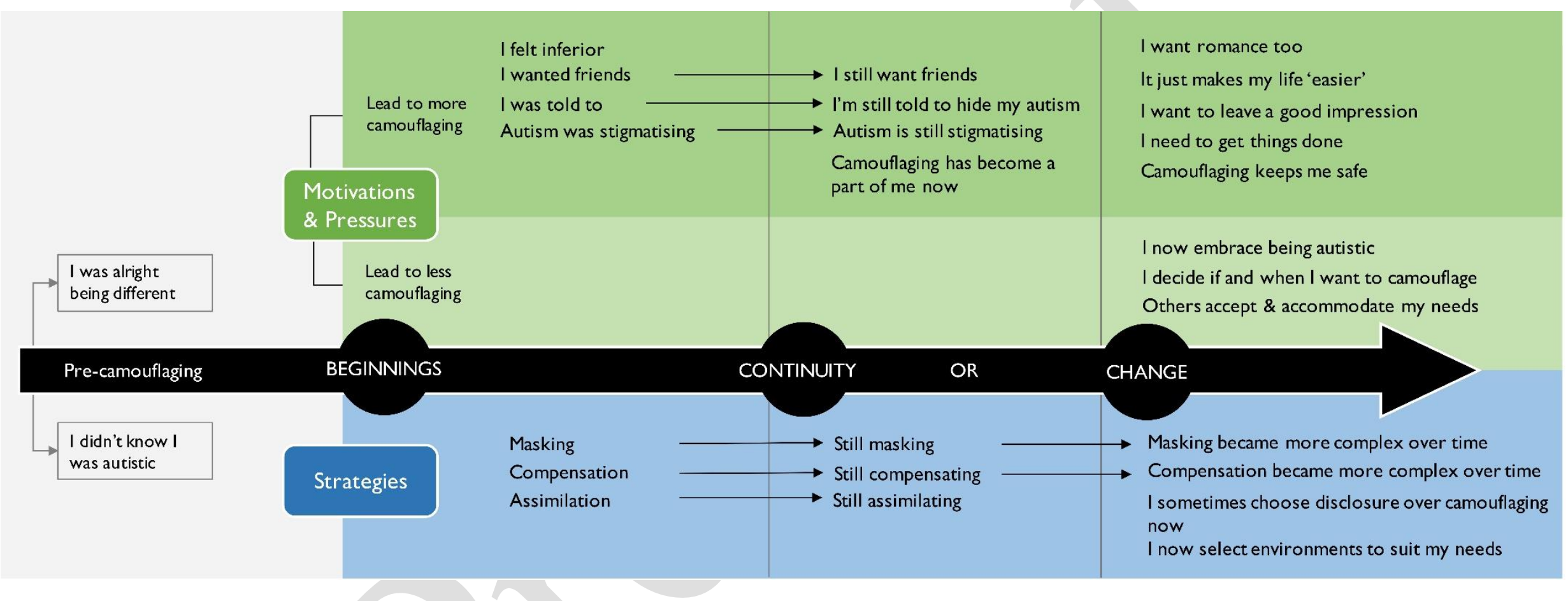

Note. The thematic map depicts themes derived across four stages of camouflaging over time (black arrow left to right: pre-camouflaging, beginnings, continuity, and change). The two domains (motivations and pressures; strategies) are represented by coloured rectangles. 


\section{Pre-Camouflaging}

I was alright being different. Most participants $(n=9)$ recalled a phase in their childhood, mostly their preschool or early primary school years when they were "alright being different". While many could recall feeling that they were in some ways different from others, most reported that they did not find their social differences impairing or concerning - "I didn't see the lack of interaction as a downside" [P02]. Others expressed a liking for being left to their passions and interests, that they "just weren't bothered at all” [P06], while another participant said that "bullying left [him] isolated, which [he] was actually pretty happy with [being left alone]" [P04].

Some participants also recalled viewing their differences positively, expressing a distaste for what their peers were interested in, and for the expectations that were placed on them to be like others. A participant explained, “I don't like being normal, doing what everyone else is doing. That's boring. So sometimes I purposely did something different, I liked that" [P09].

I didn't know I was autistic. All but two of our participants were diagnosed with autism in adolescence or adulthood. Many reported that their parents were unaware of their autism or autism in general. For some, their intense interests were understood as an indication of exceptional intellect when they were children. A late-diagnosed participant recounted, "when I was in kindergarten, my parents kind of thought that I was going to be some genius kid because I was so far ahead. [...] they saw my special interest as just another thing that maybe smart kids are interested in" [P01]. Labels such as "bookworm" or "nerd" [P04] were neutral or sometimes positively perceived and did not place pressure on our participants to change the way they were through camouflaging.

Motivations and Pressures to Camouflage

Beginnings of camouflaging: I started camouflaging because... 
I began to feel inferior. Not knowing they were autistic when they were younger meant that most of our participants did not initially understand the reasons why they felt different from others, and this was detrimental to their self-confidence and self-image over time, leaving many feeling inferior to others. While some labels in the earlier years, usually in late primary school (i.e., ages 9-12 years), were more neutral or even somewhat positive in connotation (e.g., bookworm, genius kid), participants reported increasingly feeling like they were the ones that were "abnormal", "wrong", and this was what prompted them to try to "fix" things through camouflaging.

Not to pass off as stupid, I just laughed along, like smile, laugh along, as if I understood what they're saying, but actually I didn't understand anything they were saying. [P03]

There was also a motivation to camouflage to stay out of people's attention and not stand out in order to get by. At some point, most participants said they were made aware that their behaviours were not considered "normal". They would "try not to be noticed" [P02] and attempted to behave in ways that would reduce ridicule or punishment from others. One participant diagnosed in childhood recalled that "the foundations of my camouflaging was that I didn't want people to know [I was autistic]" [P11].

I wanted friends. Most participants $(n=7)$ expressed that a desire to form and maintain friendships played a part in precipitating their earliest attempts to camouflage. Having experienced that behaving as they naturally would, "autistically", often resulted in them being excluded from social groups, many recalled beginning to hide their autistic behaviours in order to fit in and be accepted.

I wanted to join them and I wanted to be accepted. I wanted to belong. [P03]

In the same vein, seven participants reported one of their earliest social motivations to camouflage was to avoid getting ostracised by their classmates during their primary or secondary school years. Many participants recounted how they were frequently teased and 
bullied, often for behaving "autistically". Camouflaging was a strategy they employed to protect themselves, to "stop the bullying and at least have some friends so [they] wouldn't feel so depressed" [P03].

I was told to. More than half of the autistic adults in our study recalled being pressured to camouflage because adult figures in their lives (e.g., parents, teachers, therapists) told them "to be more like others" [P01]. Some participants were pressured to hide their autistic characteristics due to the negative reactions of adults if they behaved otherwise, for example, they reported receiving comments such as "don't embarrass me" [P03], "you're strange" [P05].

Autism was stigmatising. Two participants described experiencing stigma attached to being autistic in Singapore, and this seemed to be more relevant in adolescence or adulthood following diagnosis. One described how his family would "try so hard to normalise [him]... partly because of the Asian culture of [saving] face" [P03], where the colloquial term "saving face" refers to the avoidance of being humiliated or disgraced. Another suggested that "even today, people think that autism is like an attitude problem" [P06]. There was often a lack of understanding surrounding autism, and frequent collective negative attitudes about autistic people motivated them to start camouflaging.

\section{Continuity: I continue to camouflage because...}

I still want friends. Camouflaging for social connections and friendships continued to be a motivation in adult life. Some described already having a handful of friends or a best friend. Yet even with close friends, some participants also described a motivation to camouflage at times, so that "[their] friends' lives become easier", because "if I [the autistic person] rely on others to accommodate [me] too much, they [non-autistic friends] will start to move away. It's not their fault also, everyone has limited energy" [P08]. 
I'm still told to hide my autism. For a handful of participants, their families continued to remind them of socially appropriate behaviours when they were observed to display behaviours that were deemed to be 'autistic' and socially inappropriate.

And actually, the telling you what to do (by my parents) even spilled into adulthood also. Like especially before social events. [P03]

Autism is still stigmatising. As adults, most of our participants described the continuing persistent stigma attached to being autistic. One participant explained the strong emphasis on conformity in Singaporean culture and compared their experience of societal attitudes towards conformity and diversity in Singapore versus the UK, explaining that "people here [in Singapore] are more judgemental and more prejudiced. If we do not really behave in a way that's the norm, people immediately call you out on that. In the UK, they didn't really care what you do, as long as you mind your manners" [P01].

Camouflaging has become a part of me now. Camouflaging evolved to become much more natural and automatic for many adults $(n=7)$ in our study. One participant likened this process to "learning how to ride a bike, like procedural memory" [P08]. One participant explained that camouflaging had become so ingrained that it was difficult to stop or reduce despite feeling positive about being autistic.

I became overly positive of autism, to the extent that it became like, what I call, Asperger's Supremacy. [...] Actually, surprisingly I think it \{referring to Asperger Supremacy\} didn't do that much. [...] On one hand, I was thinking autism is superior but on the other hand, the old habits \{referring to camouflaging\} continued to carry through without being questioned. [P10]

Change in motivations to camouflage: I now camouflage because... 
I want romance too. Three participants reported a desire for romantic connection that emerged as a motivation for camouflaging, and all three reported camouflaging within their romantic relationships, which was especially demanding and exhausting. They "enjoyed being in the company of [their partners], but at the same time [they were] also drained" [P01].

It just makes my life 'easier'. More than half the participants explained that camouflaging makes their adult lives more manageable in some ways. Adopting a pragmatic and seemingly resigned view of why they now camouflage in their adult years, some participants explained that "it's just easier for one person to try and adapt instead of waiting for society to adapt to you" [P05]. For some participants, being their autistic selves led to more conflict, while autism disclosure led to pressures to tediously explain autism and its complexities and nuances to others. One participant shared his frustrations as such - "I cannot be explaining what autism is to every single person I meet, right? I have more productive things to do. So then, what I do is still make the decision to camouflage" [P08]. Getting by through the use of camouflaging was described to be at times less effortful and a more pragmatic option as compared to having to educate or explain one's differences to other people.

I want to leave a good impression. In adulthood, participants also shared concerns about their first impressions and reputation amongst their more distal community (e.g., acquaintances, university schoolmates). One participant shared that "the biggest reason why [they] camouflage" is that "over time [they] get more accepted by the generally large community" and considers this a "victory" won [P02]. Participants strived to build a good reputation and leave positive first impressions on others, particularly in new social settings.

Junior college was supposed to be a new start for me [...] Basically I was trying to make myself give off a positive, or at least a neutral impression, so that I don't come off as annoying at the start. [P06] 
I need to get things done. Most of our participants commonly felt the need to camouflage for practical/ functional purposes in adulthood, as more formal demands in college/ university or work emerged. Camouflaging was to them their best option for working with others and completing assignments, particularly when having to work in a team.

You need to work with people, and you need to interact with people. You can't just be yourself and expect people to be able to be okay with that. [P01]

Camouflaging keeps me safe. Two participants mentioned that, as adults, camouflaging served to prevent possible conflict and reduce autistic people's vulnerability in interpersonal situations, and also to prevent at times unwelcome sexual advances by masking and putting up a front. For example, one participant said that they engaged in camouflaging more after realising that "when others realise you take things at face value, they take advantage of you" [P06].

\section{Changes in motivations and pressures to camouflage: I now camouflage less}

\section{because...}

I now embrace being autistic. Many participants $(n=7)$ described a shift towards accepting their autistic identity. In particular, some expressed feeling "proud to be autistic" and "having better self-esteem", taking the view that "society, our family shouldn't expect us to hide, and people should just allow us to be ourselves" [P03]. This same participant noted that "this attitude" of being "proud to be autistic" only strengthened after "connecting with other autistics" in his adulthood [P03]. Another noted that "receiving my autism diagnosis was definitely the first step towards accepting myself as I am" [P05]. Participants shared an increased comfort with being different and a reduced desire to camouflage.

Accepting their autistic identity also included an affirmation of the unique and individual strengths of their autism. One example given was that "people say one thing and mean another. Technically, it's a deficit that I don't perceive those hidden meanings, but I like 
that that's how I see things. I feel that is a better way" [P09]. Rather than "always being worried about hiding" [P11] their social communication differences and difficulties, some participants described gradually choosing to invest time and energy into honing the strengths they have identified within themselves, some of which they attribute to being autistic.

I decide if and when I want to camouflage. Some participants reflected on developing increased autonomy over whether or not, when, with whom, and how they camouflage over time. One described having "learnt different ways to camouflage and then decided which [they] wanted to use" [P09]. After years of camouflaging, some others then "decided that [they] didn't want to do it anymore" [P03, P05]. In fact, some individuals talked about integrating disclosure alongside their camouflaging strategies, choosing to disclose in some situations with particular groups of people, but not disclosing and instead camouflaging in other contexts. One autistic adult shared that, although he generally preferred not to "label himself [as autistic] unless necessary" [P06] in official documents such as job applications (i.e., camouflaging in a sense), he had still privately disclosed his autism to his professors to receive accommodations in university.

Others accept and accommodate my needs. Most participants $(n=10)$ mentioned that over time they found others who were "not just accepting, but appreciated the things that come with being autistic", such as being "very logical and less emotionally charged" [P09], which led to less camouflaging with these friends. Often, friends who understood and validated their needs would plainly "tell [them] or model the way" when "[they] didn't know half the unwritten rules of social interactions" [P08] such that they felt comfortable enough not to have to camouflage as much around them.

\section{Camouflaging Strategies Over Time}

\section{Beginnings: Earliest camouflaging strategies.}


Masking was the earliest camouflaging strategy. Most participants $(n=7)$ described masking (Hull et al., 2017) as their earliest/ first strategy to camouflage their differences, even if they did not yet explicitly associate these differences with being autistic. Masking involved simple strategies initially which participants devised for themselves to follow, such as inhibiting talking about their passions or making their self-soothing stimming behaviours less visible to others. These earlier masking strategies allowed participants to "blend in subtly so no one would notice me, like putting a very big metal sheet in front of me" [P11].

Compensation. Participants reported a variety of compensatory strategies, such as showing more interest than they truly felt in social interactions or others, copying others' social or communication mannerisms, and, most commonly, purposefully regulating their eye contact, which "was one of the first things that [they] learnt" [P09]. One participant hoped "that reading the newspaper will at least give [him] some topics to talk about", which he used to strike up conversations in school [P03]. Early compensation strategies described by our participants tended to be less flexible and applied similarly across various circumstances (akin to 'shallow' compensation in the framework proposed by Livingston and Happé, 2017).

Assimilation through escape/ avoidance. Most often in a school context, almost half the participants $(n=5)$ chose at times to remove themselves from and avoid being involved in difficult social situations, which served the purpose of hiding their discomfort and distress at being in social situations.

I just took a lot of offs [days off - sick leave] from school. But I'm just pretending. Because I was just too tired of trying to do this day in, day out. Trying to face people day in, day out. [P06]

\section{Continuity: Early camouflaging strategies I continue to use.}

Masking. Masking persisted into later phases of life particularly in formal work contexts, but also in social contexts. Participants described continuing to mask regularly, such 
as by inhibiting stimming behaviours or making efforts to speak less extensively about their interests.

When I feel the urge to talk, then I try not to talk, I try to sit there and do my own work. [P03]

Compensation. Participants described growing more accustomed to using different compensation strategies, and that their compensation strategies became more complex, less effortful, and more flexible over time.

When social interaction comes I will always try to see how the person talks and behaves. Their thinking process also. I dive right deep into that. [...] And react to it accordingly. I have developed this over time because I like to process a lot. How can I avoid this in the future? Is it something that I have done, or something about me that they are just picking out? So that allowed me to evaluate people more, how they behave, and react accordingly. [P11]

Assimilation through escape/ avoidance. Some participants continued to use escape or avoidance as a way to conceal and manage their discomfort and to cope with the toll of trying to fit in and constantly hide their autism in social interactions, for example, "going to the toilet and just staying inside for 15-20 minutes to recharge" [P01]. Some avoided social situations as they were aware of their emotional needs, and took intentional breaks from social interaction when needed, minimising the visibility of their differences to others by preventing a meltdown.

\section{Change: How my camouflaging strategies developed or changed over time.}

Masking became more complex over time. While earlier masking involved hiding oneself by fading into the background, more complex masking strategies emerged in adulthood. Instead of scripting responses, for example, one autistic adult described adjusting personas to suit various contexts - he was "flamboyant" and outgoing with close friends but assumed 
another distinct personality with colleagues and acquaintances, "a more aloof kind of persona so they don't push themselves too much on you" [P04].

Compensation became more complex over time. About a third of our participants explicitly talked about using a deliberate combination of various sources of information (e.g., logic, tone of voice) to develop and employ 'deeper' alternative cognitive routes to process and understand social situations. Participants also recounted using what they recognised as strengths of being an autistic individual to facilitate the use of some compensation strategies. One participant shared that "I notice patterns and use these patterns to tell my friends about their thinking patterns and insecurities, and that wins friends" [P06]. Identifying patterns to make sense of social behaviour and others' thoughts, "like a formula" [P09], was also commonly mentioned, suggesting deeper and more sophisticated compensation strategies over time.

I sometimes choose disclosure over camouflaging now. In direct opposition to camouflaging, disclosing one's autistic diagnosis was a change that emerged only in adulthood for our participants, although it should be noted that many were diagnosed late in adolescence or adulthood and it is possible that a different pattern could be found for people diagnosed as children, who may be more used to disclosing their autism earlier on in their lives. Five autistic adults talked about opting for disclosure, and they all shared positive outcomes of disclosing their autistic identity, such as increased understanding and accommodation of their needs amongst friends and colleagues. One participant [P04] decided to be honest about being autistic at job interviews so that he would not "have to worry about everything" and found that "the interviews actually went okay". He took the time to explain his need for support in social situations sometimes, such as "[needing] to set up some sort of structure for collaborative work and make sure everyone sticks to the structure". Another participant worried about the outcome of letting classmates know of their diagnosis, but shared their surprise in the following quote: 
I am so glad that my psychologist encouraged me to tell the class I was autistic because they went out of their way to include me in everything. [...] They were very patient, to the point where I jokingly called them my "fellowship"... they were literally a case of “safe space”. [P07]

I now select environments to suit my needs. Lastly, many participants also described more carefully selecting their social or formal environments now to better suit their needs, playing to their strengths rather than their difficulties. This reduced the need to camouflage in these environments, as participants were often more comfortable to be their authentic selves. One participant concluded that "a research job is probably one of the few places you can wear more casual clothes", which suited his sensory needs [P05]. Another learnt to "negotiate with [his] boss not to get him to front too many customers", such that he would do so "only when there was no one else to do it" [P10].

\section{Discussion}

This study aimed to explore the beginnings, continuity, and change in camouflaging motivations and strategies through in-depth qualitative interviews that invited participants to recall and reflect on their camouflaging experiences throughout their lives.

\section{Beginnings: The Emergence of Early Camouflaging Behaviours}

Often during preschool or early primary school years, most participants described initially not feeling a need or pressure to camouflage, with some being unaware of their differences, preferring to spend time alone, or not initially facing pressures from their caregivers to adjust their behaviours, as their social differences at the time were perceived by their parents as personality differences or as a sign of intelligence and superiority.

Yet, for many autistic adults, motivations to blend in, which were largely social in nature, soon emerged during the later primary or early secondary school years and settings. In contrast to their earlier comfort with being different, many participants began "pretending to be 
normal" as feelings of inferiority to their peers developed. Participants' accounts suggested that these earliest emerging motivations to camouflage were linked to a negative self-identity they developed as a consequence of distressing and adverse social experiences. They described being bullied and ostracised because of their social differences or for their seemingly "unusual" interests, sharing experiences common to many other autistic people (e.g., Cappadocia et al., 2012; Griffiths et al., 2019). Parents' and teachers' constant urging to "be more like" their peers and to act and interact like them, were commonly reported by participants. Drawing from the biopsychosocial conceptualisation of health and disease (see Bolton \& Gillett, 2019; Engel, 1977), this pathologizing and stigmatisation of autism and autistic differences, alongside consistent negative responses from others, can negatively impact an autistic person's construction of their self-identity (Humphrey \& Lewis, 2008; Zahavi, 2010, as cited in Huang et al., 2017). Our participants reported internalising negative labels, such as being "stupid" and "abnormal", since childhood. As these experiences in childhood increasingly signalled to the autistic child that their social or behavioural differences were a negative aspect of their identity, camouflaging seemed to emerge as a "remedy" to hide these "negative" differences, to manage or reduce social rejection and its associated emotional distress. Indeed, camouflaging can be conceptualised as a stigma-management strategy involving the concealment of one's autistic identity in response to autism-related stigma (Botha et al., 2020; Cage \& Troxell-Whitman, 2019; Pearson \& Rose, 2021; Perry et al., 2021). The emergence of the earliest camouflaging behaviours is represented by progression A in Figure 2. 


\section{Figure 2}

Integrated Trajectories of Camouflaging Behaviour Over Time and Their Associated Psychosocial Influences based on our participants' interview data

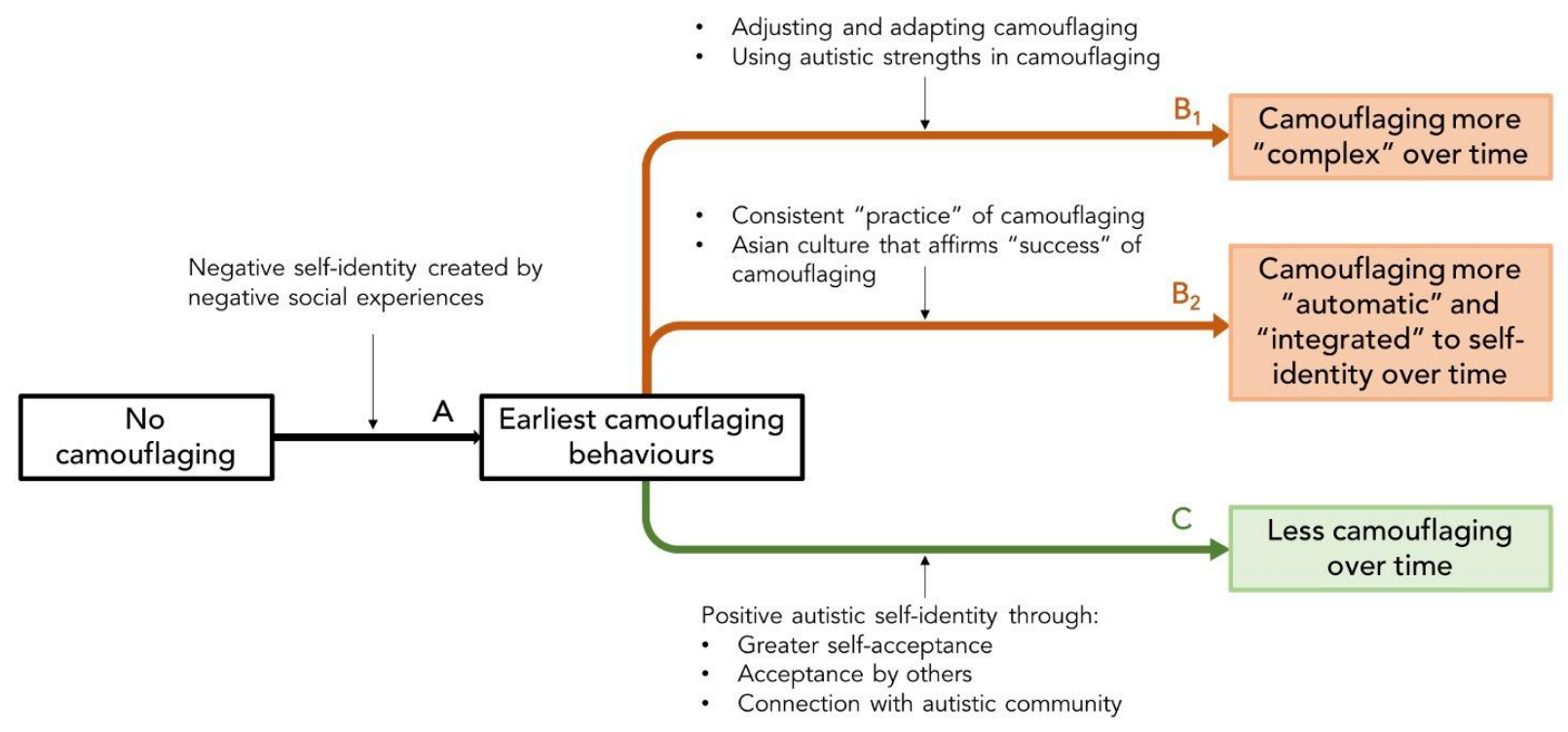

Camouflaging Continued and Evolved Over Time, Becoming More "Complex", "Deeper" and More "Integrated" to an Autistic Adult's Identity

Camouflaging Became More Complex and Deeper Over Time

Our participants told us that masking, compensation, and assimilation strategies emerged as their earliest camouflaging strategies, with masking being the most common early strategy; these strategies continued but also evolved over time for some of our participants to become “deeper" and more complex (see Trajectory $B_{1}$ in Figure 2). Some autistic adults learnt to use their autistic strengths, for example, acute visual processing, sensitivity to patterns, and good recall, to make sense of novel social situations over time, to more accurately interpret others' social cues, create social rules and systems, and to adjust their autistic behaviours accordingly. This process of learning to camouflage by using autistic strengths was viewed positively by participants in our study; developing a more sophisticated and flexible form of camouflaging was often coupled with more perceived "success" of camouflaging over time and a feeling of camouflaging becoming "easier" and less effortful. 
Autistic adults in a study by Schneid and Raz (2020) also considered that a "deeper" form of camouflaging - involving seeking and understanding the meaning of social interactions - was better than "imitation" as in shallow camouflaging (e.g., laughing when others laugh without understanding why), which to them represented a coerced way of blending into the social environment. Our findings are consistent with those of Schneid and Raz and suggest that learning to identify and use autistic strengths may facilitate camouflaging with deeper understanding, and consequently could be viewed as a useful social "skill" that could be acquired over time. It is possible that such an approach to camouflaging may be less taxing and potentially less linked to autistic burnout and poor mental wellbeing, although this hypothesis needs to be empirically investigated.

\section{Camouflaging Became More “Integrated” to an Autistic Adult's Self-Identity Over Time}

Autistic adults in our study often considered their earliest camouflaging attempts as confusing, effortful, and largely prescribed by authority figures, similar to experiences shared by other autistic adults in the literature (e.g., Bargiela et al., 2016; Hull et al., 2017). At the same time, many of our participants ( 7 of 11) also described camouflaging as becoming more integrated into their autistic self-identity over time, as detailed by the theme "Camouflaging has become a part of me now" (see Trajectory $B_{2}$ in Figure 2). Camouflaging had become "natural" and "automatic", resonating with experiences of autistic adults in other qualitative studies (Bradley et al., 2021; Cage \& Troxell-Whitman, 2019; Cook, Crane, Bourne, et al., 2021; Miller et al., 2021). Many of our participants viewed this as an adaptive change from their earlier much more pressurising and taxing camouflaging efforts. One participant put it this way, “camouflaging is not about masking or pretending, [...] it's just a natural me being me in a way that fits in with other people, but without losing my true self. It's something that neurotypicals attain at a very early age". This shares a striking similarity to impression management by nonautistic people, which involves adjusting self-presentations in different social contexts and for different social purposes, but without the sense of compromising one's core "true" sense of self, 
a view that has been expressed by other autistic adults (Schneid \& Raz, 2020), however empirical investigations attempting to explore overlaps and differences between camouflaging and impression management are currently lacking and needed.

However, some autistic adults may not experience automatisation of camouflaging behaviours and may instead find it a constant struggle to know how to camouflage and do so successfully (Cook, Crane, Bourne, et al., 2021; Livingston et al., 2019). Camouflaging may even become increasingly difficult with age (Miller et al., 2021), and some autistic individuals may have a reduced capacity to camouflage due to experiences of autistic burnout from accumulated life stressors (Higgins et al., 2021; Raymaker et al., 2020). Alternatively, for those who are able to camouflage automatically and unconsciously, this could potentially lead to a loss of sense of self when their 'masked' and true selves become so intricately intertwined with one another (Miller et al., 2021). Integrating the above studies' findings with our own, there appears to be a large variation in autistic adults' camouflaging experiences and attitudes, with some viewing camouflaging as akin to impression management and able to get better at it with practice, but others finding it a continual challenge and/or detrimental to their identity. Such variation may relate to the extent to which one engages in camouflaging, the type of camouflaging (e.g., superficial social behaviours, hiding fundamental aspects of oneself), or the outcome (e.g., degree of success) of camouflaging attempts.

Of note, all our participants were of Asian ethnicity (10 of 11 were Asian Chinese) and had resided in Singapore for most of their lives. According to Hofstede's (2011) Model of Cultural Dimensions, the Singaporean society subscribes to a largely collectivist culture (“Hofstede Insights", n.d., para. 3), where people identify foremostly as belonging to an ingroup (e.g., country, family, organisation), and where society values maintaining group harmony over one's individuality, such that the transgression of norms can lead to feelings of shame. Growing up and being socialised in a more collectivist society, it is perhaps not surprising that camouflaging was viewed by several autistic adults in our study as a skill to 
"improve", since this may have led to social reward or reduced social rejection (i.e., being "good" at camouflaging was beneficial to them). While this cultural context may have contributed to participants considering some camouflaging behaviours as adaptive and necessary, it is possible that sociocultural pressures have bred, to some extent, internalised ableism for some of our participants (Silverman, 2019) - that they have internalised believing that social acceptance is possible only for autistic people who are able to "fix" themselves to fit into "typical" society's moulds. As camouflaging is often a socially motivated behaviour, exploring broader sociocultural influences in motivations for camouflaging across different societies/ countries will likely be a worthwhile future research endeavour in better understanding the more distal sociocultural correlates of camouflaging.

\section{Camouflaging Reduced Over Time}

Lastly, a key change in adulthood for some participants was a shift towards choosing disclosure of their autism over camouflaging at times and experiencing reduced pressure to camouflage (see Trajectory C in Figure 2; see also Hull et al., 2018, who also reported a negative association between age and CAT-Q scores, and Livingston et al. (2019), who report on a subgroup of autistic people who use fewer compensatory strategies over time). Reduced camouflaging over time may also be related to autistic adults more actively seeking out environments that are more suited to their needs and authentic selves, akin to accommodation described by Livingston and colleagues (2020). When an autistic person communicates their inner differences and needs to others, while clarifying others' misconceptions and misunderstandings, their autistic differences become legitimised, paving the way for them and for others to accept autism as a different identity, without stigma (Schneid and Raz, 2020).

Many participants described that this change was linked to a shift towards embracing a more positive self-identity over time - as captured by the theme "I now embrace being autistic" - in addition to increased appreciation of autism as diversity not deficit, greater self-compassion, and re-evaluating others' standards of social "normalcy". This is consistent with Cage and 
Troxell-Whitman's (2020) finding that a stronger autistic identity was linked to greater autism disclosure, which in turn led to reduced engagement in camouflaging. Participants in our study did not always explicitly detail how this positive autistic self-identity developed over time, although parts of this process were attributed to (a) individual psychological changes, and (b) positive social experiences that increasingly reinforced their self-worth as an autistic person. Relating to individual psychological changes, some participants shared that obtaining their autism diagnosis in later life was a key experience that helped them begin a gradual process of greater self-acceptance. All but two of our participants were late-diagnosed in adolescence or adulthood, and for a majority, their diagnosis marked the beginning of understanding the reasons for, and accepting, their differences, and then also understanding and identifying with the strengths of being autistic, in line with the process of identity-building described in recent studies of late-diagnosed autistic women (Bargiela et al., 2016; Leedham et al., 2019). In relation to positive social experiences, about half of our participants described finding an "inner circle" of friends - people who knew of their diagnosis, accepted them for who they were, and with whom participants did not feel the pressure or need to camouflage. Indeed, autistic people have reported developing a positive sense of identity due to their ability to maintain trusting and authentic relationships with others (Cribb et al., 2019). Furthermore, all participants in our study who had disclosed being autistic generally reported obtaining accommodations and supports at school or at work, and increased emotional support and understanding of their differences and difficulties by peers. This fits with findings from a study by Sasson and Morrison (2019) that non-autistic people's first impressions of autistic adults improved with diagnostic disclosure and with increased autism knowledge. Overall, our findings echo recent qualitative studies that suggest that the transition to reduced camouflaging over time stems from a process of selfacceptance (aided by an autism diagnosis for some) and other-acceptance (Bradley et al., 2021; Cook, Crane, Bourne, et al., 2021). The quote by participant [P07] presented earlier that talking to their classmates about being autistic resulted in them becoming a "fellowship" and a "safe 
space" for them aptly sums up the potential positive impact an autistic person's surrounding community can have.

\section{Strengths and Limitations of the Present Study}

Our participants formed a self-selected group of autistic adults; while many autistic adults often experience high rates of mental health difficulties and distress (Hollocks et al., 2019; Cassidy et al., 2018), individuals who reported having experienced significant acute emotional distress, or thoughts of harming themselves or others currently or in the past six months were not included in this study. Most of our participants were also ethnically Chinese (the majority ethnic group in Singapore), in tertiary education or currently/ previously employed. Lessons learnt from this sample and their social experiences speak to how pressures to camouflage may be reduced and/or how the personal costs of camouflaging may be attenuated, but may not be directly generalisable to other autistic people in different contexts. Future camouflaging research should include autistic individuals with more diverse social and emotional experiences to further shed light on important risk factors that may predict poorer camouflaging outcomes, such as experiencing elevated depressive or anxiety symptoms and/or suicidal ideation, or a diminished sense of self.

To the best of our knowledge, our study was the first to focus specifically on investigating the beginnings, continuity, and change in camouflaging through inviting adults to recall and describe in detail their earliest camouflaging memories and the trajectory of their camouflaging behaviours over time. However, our adult participants at times found it difficult to clearly recall childhood experiences of camouflaging and to explicitly describe underlying personal characteristics or processes involved in the apparent changes in camouflaging behaviour or attitudes. There may also be some partiality when recalling potentially distressing events surrounding social rejection or "failure" due to self-enhancement tendencies (Lalande \& Bonanno, 2011) that may bias participants' accounts of past camouflaging experiences. Future research will therefore benefit from more direct interviews with children and young people with 
subsequent follow-up designs, to further build our understanding of how camouflaging and its impact may evolve over time, and to elucidate the associated influences of these changes. Our interview methodology was able to tap on what autistic adults thought about their camouflaging efforts, but of course less conscious camouflaging (e.g., unconscious social mimicry) would not be reported - discrepancy approaches (comparing social behaviour and underlying sociocognitive processing) have been suggested as an alternative method to study camouflaging and compensation (e.g., Livingston et al, 2019).

\section{Implications and Recommendations for Clinical Practice and Social Change}

Our results extend previous research (e.g., Cage \& Troxell-Whitman, 2020) by showing that building a strong and positive autistic identity may reduce autistic people's felt pressure to camouflage and may afford autistic people a greater sense of autonomy over whether, when, with whom, and for which purposes, they may choose to engage (or not) in camouflaging strategies (see also theme "I decide if and when I want to camouflage"). Having a positive autistic identity also appeared to be related to our participants' experiences of self-reported greater comfort and ease with one's experiences of camouflaging, and it would be important to further and more systematically investigate the relationship between a stronger positive autistic identity and whether this may buffer from the negative impacts of camouflaging. Timely psychosocial support for autistic people of all ages, focusing on navigating a new autistic identity, identifying and building trusting and authentic relationships with others, and engaging constructively and helpfully with the autistic community for support and validation postdiagnosis, may facilitate a more positive self-identity and strengthen autism self-acceptance (Cage et al., 2018). This then reduces the pressure to camouflage, and/or may protect against experiencing poorer mental health, burnout, or a loss of self when having to camouflage in certain contexts.

Yet, research has shown that people living with concealable stigmatised identities sometimes still express a preference to continue hiding their identity to secure acceptance, 
although hiding a stigmatised identity can, conversely, reduce feelings of authenticity and belonging (Davidson \& Henderson, 2010; Newheiser \& Barreto, 2014). This captures the dissonance and dilemma that autistic people may face when "choosing" between camouflaging and disclosure. Autistic people should be aided to identify and select individuals, groups, and communities of people who would accept and support them when made aware of their diagnosis, which could reduce the commonly felt fear and uncertainty of the outcomes of disclosure (Huws \& Jones, 2008; Johnson \& Joshi, 2014). In particular, this could involve facilitating their relationships with autistic peers and facilitating introduction to an autistic community, given that many autistic individuals report being more comfortable and authentic around other autistic people (Crompton et al., 2020). Psychosocial supports and services could focus on supporting autistic people to strategically, yet accurately and confidently, present themselves as autistic to others, rather than focusing merely on skills-based interventions (e.g., social skills training) as many interventions currently do (Kandalaft et al., 2013; Nuernberger et al., 2013). In this way, it may be possible to use both disclosure and camouflaging strategies as impression management at different times to educate and increase others' understanding of autism and form authentic connections, while retaining a strong sense of identity as an autistic person.

\section{Summary and Conclusions}

Psychological and social experiences shape how autistic individuals perceive their autistic identity, and their consequent motivation to mask being autistic (or not) through camouflaging strategies. Although initial relational and functional motivations to camouflage still remained, we observed a recalled trajectory of reduced motivation to camouflage over time in our participants' narratives due to greater self-acceptance, and through acceptance, support, and accommodations from others. Our findings suggest that some autistic people use camouflaging strategies in conjunction with disclosure, depending on the social context in which they find themselves, to gain the utility of camouflaging while keeping costs to one's 
self-identity and well-being low. We hope that this study will prompt future age cohort comparisons and prospective longitudinal research on how social camouflaging starts, continues, and changes over time. Such work can help inform psychological and social efforts directed towards alleviating the pressures and demands on autistic individuals to camouflage. These efforts may need to focus on reducing autism stigma, growing acceptance, tailoring support, and encouraging accommodation of the needs of autistic people in their families, schools, community, and work settings, as well as strengthening autistic self-identity in psychological work with autistic people from childhood onwards and throughout their lives. 


\section{References}

American Psychiatric Association. (2013). Diagnostic and statistical manual of mental disorders (5th ed.).

Bargiela, S., Steward, R., \& Mandy, W. (2016). The experiences of late-diagnosed women with autism spectrum conditions: An investigation of the female autism phenotype. Journal of autism and developmental disorders, 46(10), 3281-3294.

Beck, J. S., Lundwall, R. A., Gabrielsen, T., Cox, J. C., \& South, M. (2020). Looking good but feeling bad: "Camouflaging" behaviours and mental health in women with autistic traits. Autism, 24(4), 809-821. https://doi.org/10.1177/1362361320912147

Begeer, S., Mandell, D., Wijnker-Holmes, B., Venderbosch, S., Rem, D., Stekelenburg, F., \& Koot, H. M. (2013). Sex differences in the timing of identification among children and adults with autism spectrum disorders. Journal of autism and developmental disorders, 43(5), 1151-1156.

Bernardin, C. J., Lewis, T., Bell, D., \& Kanne, S. (2021). Associations between social camouflaging and internalising symptoms in autistic and non-autistic adolescents. Autism, 1362361321997284. https://doi.org/10.1177/1362361321997284

Bernardin, C. J., Mason, E., Lewis, T., \& Kanne, S. (2021). "You must become a chameleon to survive": Adolescent experiences of camouflaging. Journal of Autism and Developmental Disorders. https://doi.org/10.1007/s10803-021-04912-1

Bolton, D., \& Gillett, G. (2019). The biopsychosocial model of health and disease: New philosophical and scientific developments. Cham: Springer International Publishing.

Botha, M., Dibb, B., \& Frost, D. M. (2020). "Autism is me": An investigation of how autistic individuals make sense of autism and stigma. Disability \& Society, 1-27. https://doi.org/10.1080/09687599.2020.1822782

Bradley, L., Shaw, R., Baron-Cohen, S., \& Cassidy, S. (2021). Autistic adults' experiences of 
camouflaging and its perceived impact on mental health. Autism in Adulthood. Advance online publication. https://doi.org/10.1089/aut.2020.0071

Bronfenbrenner, U. (1994). Ecological models of human development. Readings on the development of children, 2(1), 37-43.

Cage, E., Di Monaco, J., \& Newell, V. (2018). Experiences of autism acceptance and mental health in autistic adults. Journal of autism and developmental disorders, 48(2), 473484.

Cage, E., \& Troxell-Whitman, Z. (2019). Understanding the reasons, contexts and costs of camouflaging for autistic adults. Journal of Autism and Developmental Disorders, 49(5), 1899-1911.

Cage, E., \& Troxell-Whitman, Z. (2020). Understanding the relationships between autistic identity, disclosure, and camouflaging. Autism in Adulthood, 2(4), 334-338. https://doi.org/10.1089/aut.2020.0016

Cappadocia, M. C., Weiss, J. A., \& Pepler, D. (2012). Bullying experiences among children and youth with autism spectrum disorders. Journal of autism and developmental disorders, 42(2), 266-277.

Cassidy, S., Bradley, L., Shaw, R., \& Baron-Cohen, S. (2018). Risk markers for suicidality in autistic adults. Molecular Autism, 9(1), 42.Cassidy, S. A., Gould, K., Townsend, E., Pelton, M., Robertson, A. E., \& Rodgers, J. (2020). Is camouflaging autistic traits associated with suicidal thoughts and behaviours? Expanding the interpersonal psychological theory of suicide in an undergraduate student sample. Journal of Autism and Developmental Disorders, 50(10), 3638-3648. https://doi.org/10.1007/s10803019-04323-3

Cook, J., Crane, L., Bourne, L., Hull, L., \& Mandy, W. (2021). Camouflaging in an everyday social context: An interpersonal recall study. Autism, 25(5), 1444-1456. https://doi.org/10.1177/1362361321992641 
Cook, J., Crane, L., Hull, L., Bourne, L., \& Mandy, W. (2021). Self-reported camouflaging behaviours used by autistic adults during everyday social interactions. Autism. Advance online publication. https://doi.org/10.1177/13623613211026754

Cook, A., Ogden, J., \& Winstone, N. (2018). Friendship motivations, challenges and the role of masking for girls with autism in contrasting school settings. European Journal of Special Needs Education, 33(3), 302-315. https://doi.org/10.1080/08856257.2017.1312797

Corbett, B. A., Schwartzman, J. M., Libsack, E. J., Muscatello, R. A., Lerner, M. D., Simmons, G. L., \& White, S. W. (2021). Camouflaging in autism: Examining sex-based and compensatory models in social cognition and communication. Autism Research, 14(1), 127-142. https://doi.org/10.1002/aur.2440

Crompton, C. J., Hallett, S., Ropar, D., Flynn, E., \& Fletcher-Watson, S. (2020). 'I never realised everybody felt as happy as I do when I am around autistic people': A thematic analysis of autistic adults' relationships with autistic and neurotypical friends and family. Autism, 24(6), 1438-1448. https://doi.org/10.1177/1362361320908976

Cribb, S., Kenny, L., \& Pellicano, E. (2019). 'I definitely feel more in control of my life': The perspectives of young autistic people and their parents on emerging adulthood. Autism, 23(7), 1765-1781.

Davidson, J., \& Henderson, V. L. (2010). 'Coming out' on the spectrum: autism, identity and disclosure. Social \& Cultural Geography, 11(2), 155-170.

Dean, M., Harwood, R., \& Kasari, C. (2017). The art of camouflage: Gender differences in the social behaviors of girls and boys with autism spectrum disorder. Autism, 21(6), 678689.

Elmose, M. (2016). A conceptual framework for understanding characteristics of selfawareness associated with autism spectrum disorder. Scandinavian Journal of Child and Adolescent Psychiatry and Psychology, 4(3), 109-114. 
Engel, G. L. (1977). The need for a new medical model: a challenge for biomedicine. Science, 196(4286), 129-136.

Griffiths, S., Allison, C., Kenny, R., Holt, R., Smith, P., \& Baron-Cohen, S. (2019). The Vulnerability Experiences Quotient (VEQ): A study of vulnerability, mental health and life satisfaction in autistic adults. Autism Research, 12(10), 1516-1528.

Gould, J. (2017). Towards understanding the under-recognition of girls and women on the autism spectrum. Autism, 21(6), 703-705.

Halsall, J., Clarke, C., \& Crane, L. (2021). “Camouflaging” by adolescent autistic girls who attend both mainstream and specialist resource classes: Perspectives of girls, their mothers and their educators. Autism. https://doi.org/10.1177/13623613211012819

Higgins, J. M., Arnold, S. R., Weise, J., Pellicano, E., \& Trollor, J. N. (2021). Defining autistic burnout through experts by lived experience: Grounded Delphi method investigating \#AutisticBurnout. Autism. Advance online publication. https://doi.org/10.1177/13623613211019858

Hoekstra, R. A., Vinkhuyzen, A. A., Wheelwright, S., Bartels, M., Boomsma, D. I., BaronCohen, S., ... \& Van Der Sluis, S. (2011). The construction and validation of an abridged version of the autism-spectrum quotient (AQ-Short). Journal of autism and developmental disorders, 41(5), 589-596.

Hofstede, G. (2011). Dimensionalising cultures: The Hofstede model in context. Online readings in psychology and culture, 2(1), 2307-0919.

Hollocks, M. J., Lerh, J. W., Magiati, I., Meiser-Stedman, R., \& Brugha, T. S. (2019). Anxiety and depression in adults with autism spectrum disorder: A systematic review and metaanalysis. Psychological Medicine, 49(4), 559-572. https://doi.org/10.1017/S0033291718002283

Howlin, P., \& Magiati, I. (2017). Autism spectrum disorder: Outcomes in adulthood. Current Opinion in Psychiatry, 30(2), 69-76. 
Huang, A. X., Hughes, T. L., Sutton, L. R., Lawrence, M., Chen, X., Ji, Z., \& Zeleke, W. (2017). Understanding the self in individuals with autism spectrum disorders (ASD): A review of literature. Frontiers in psychology, 8, 1422.

Hull, L., Levy, L., Lai, M. C., Petrides, K. V., Baron-Cohen, S., Allison, C., Smith, P., \& Mandy, W. (2021). Is social camouflaging associated with anxiety and depression in autistic adults? Molecular Autism, 12(1), 13. https://doi.org/10.1186/s13229-02100421-1

Hull, L., Mandy, W., Lai, M. C., Baron-Cohen, S., Allison, C., Smith, P., \& Petrides, K. V. (2018). Development and validation of the camouflaging autistic traits questionnaire (CAT-Q). Journal of Autism and Developmental Disorders, 49(3), 819-833.

Hull, L., Petrides, K. V., Allison, C., Smith, P., Baron-Cohen, S., Lai, M. C., \& Mandy, W. (2017). "Putting on my best normal": social camouflaging in adults with autism spectrum conditions. Journal of autism and developmental disorders, 47(8), 2519-2534.

Hull, L., Petrides, K. V., \& Mandy, W. (2021). Cognitive predictors of self-reported camouflaging in autistic adolescents. Autism Research, 14(3), 523-532. https://doi.org/10.1002/aur.2407 Humphrey, N., \& Lewis, S. (2008). Make me normal' The views and experiences of pupils on the autistic spectrum in mainstream secondary schools. Autism, 12(1), 23-46.

Huws, J. C., \& Jones, R. S. (2008). Diagnosis, disclosure, and having autism: An interpretative phenomenological analysis of the perceptions of young people with autism. Journal of Intellectual and Developmental Disability, 33(2), 99-107.

Johnson, T. D., \& Joshi, A. (2014). Disclosure on the spectrum: Understanding disclosure among employees on the autism spectrum. Industrial and Organisational Psychology, $7(2), 278-281$.

Kandalaft, M. R., Didehbani, N., Krawczyk, D. C., Allen, T. T., \& Chapman, S. B. (2013). Virtual reality social cognition training for young adults with high-functioning autism. 
Journal of autism and developmental disorders, 43(1), 34-44.

Kuo, A. A., Crapnell, T., Lau, L., Anderson, K. A., \& Shattuck, P. (2018). Stakeholder perspectives on research and practice in autism and transition. Pediatrics, 141(Suppl 4), S293-S299.

Lalande, K. M., \& Bonanno, G. A. (2011). Retrospective memory bias for the frequency of potentially traumatic events: A prospective study. Psychological Trauma: Theory, Research, Practice, and Policy, 3(2), 165.

Lai, M. C., Lombardo, M. V., Ruigrok, A. N., Chakrabarti, B., Auyeung, B., Szatmari, P., ... \& MRC AIMS Consortium. (2017). Quantifying and exploring camouflaging in men and women with autism. Autism, 21(6), 690-702.

Leedham, A., Thompson, A. R., Smith, R., \& Freeth, M. (2020). 'I was exhausted trying to figure it out': The experiences of females receiving an autism diagnosis in middle to late adulthood. Autism, 24(1), 135-146.

Lehman, B. J., David, D. M., \& Gruber, J. A. (2017). Rethinking the biopsychosocial model of health: Understanding health as a dynamic system. Social and Personality Psychology Compass, 11(8), e12328-n/a. doi:10.1111/spc3.12328

Livingston, L. A., \& Happé, F. (2017). Conceptualising compensation in neurodevelopmental disorders: Reflections from autism spectrum disorder. Neuroscience \& Biobehavioral Reviews, 80, 729-742.

Livingston, L. A., Shah, P., \& Happé, F. (2019). Compensatory strategies below the behavioural surface in autism: a qualitative study. The Lancet Psychiatry, 6(9), 766777.

Livingston, L. A., Shah, P., Milner, V., \& Happé, F. (2020). Quantifying compensatory strategies in adults with and without diagnosed autism. Molecular autism, 11(1), 15.

Lord, C., Bishop, S., \& Anderson, D. (2015). Developmental trajectories as autism phenotypes. American Journal of Medical Genetics. Part C, Seminars in Medical Genetics, 169(2), 
198-208.

Lord, C., Brugha, T. S., Charman, T., Cusack, J., Dumas, G., Frazier, T., ... \& Pickles, A. (2020). State, MW Autism spectrum disorder. Nat. Rev. Dis. Primers, 6, 1-23.

Magiati, I., Tay, X. W., \& Howlin, P. (2014). Cognitive, language, social and behavioural outcomes in adults with autism spectrum disorders: A systematic review of longitudinal follow-up studies in adulthood. Clinical psychology review, 34(1), 73-86.

Miller, D., Rees, J., \& Pearson, A. (2021). "Masking is life": Experiences of masking in autistic and non-autistic adults. Autism in Adulthood. https://doi.org/10.1089/aut.2020.0083

Milton, D. E. (2012). On the ontological status of autism: the 'double empathy problem'. Disability \& Society, 27(6), 883-887.

Newheiser, A. K., \& Barreto, M. (2014). Hidden costs of hiding stigma: Ironic interpersonal consequences of concealing a stigmatised identity in social interactions. Journal of Experimental Social Psychology, 52, 58-70.

Nuernberger, J. E., Ringdahl, J. E., Vargo, K. K., Crumpecker, A. C., \& Gunnarsson, K. F. (2013). Using a behavioral skills training package to teach conversation skills to young adults with autism spectrum disorders. Research in Autism Spectrum Disorders, 7(2), 411-417.

Pearson, A., \& Rose, K. (2021). A conceptual analysis of autistic masking: Understanding the narrative of stigma and the illusion of choice. Autism in Adulthood, 3(1), 52-60. https://doi.org/10.1089/aut.2020.0043

Perry, E., Mandy, W., Hull, L., \& Cage, E. (2021). Understanding camouflaging as a response to autism-related stigma: A social identity theory approach. Journal of Autism and Developmental Disorders. https://doi.org/10.1007/s10803-021-04987-w

Raymaker, D. M., Teo, A. R., Steckler, N. A., Lentz, B., Scharer, M., Delos Santos, A., Kapp, S. K., Hunter, M., Joyce, A., \& Nicolaidis, C. (2020). "Having all of your internal resources exhausted beyond measure and being left with no clean-up crew": Defining 
autistic burnout. Autism in Adulthood, 2(2), 132-143.

Sasson, N. J., Faso, D. J., Nugent, J., Lovell, S., Kennedy, D. P., \& Grossman, R. B. (2017). Neurotypical peers are less willing to interact with those with autism based on thin slice judgments. Scientific Reports, 7(1), 40700.

Sasson, N. J., \& Morrison, K. E. (2019). First impressions of adults with autism improve with diagnostic disclosure and increased autism knowledge of peers. Autism, 23(1), 50-59.

Schneid, I., \& Raz, A. E. (2020). The mask of autism: Social camouflaging and impression management as coping/normalisation from the perspectives of autistic adults. Social Science \& Medicine, 248, 112826.

Schroeder, J. H., Cappadocia, M. C., Bebko, J. M., Pepler, D. J., \& Weiss, J. A. (2014). Shedding light on a pervasive problem: A review of research on bullying experiences among children with autism spectrum disorders. Journal of autism and developmental disorders, 44(7), 1520-1534.

Silverman, A. (2019). Contending with ableism from internalised ableism to collective action. Ableism: The Causes and Consequences of Disability Prejudice, 220-265.

Tierney, S., Burns, J., \& Kilbey, E. (2016). Looking behind the mask: Social coping strategies of girls on the autistic spectrum. Research in Autism Spectrum Disorders, 23, 73- 83. 


\section{Appendix 1}

Table 1

Sample Questions of Main Interview Segments

\begin{tabular}{|c|c|c|}
\hline Interview segment & Opening question & Sample follow-up questions \\
\hline $\begin{array}{l}\text { Part 1: Beginnings and } \\
\text { earliest memories of } \\
\text { camouflaging }\end{array}$ & $\begin{array}{l}\text { Tell us about your earliest } \\
\text { memories of you } \\
\text { camouflaging. }\end{array}$ & $\begin{array}{l}\text { A. Where/what were you } \\
\text { doing at the time? Who } \\
\text { were you with? } \\
\text { B. More generally, were } \\
\text { your earlier attempts to } \\
\text { camouflage with } \\
\text { specific people? Who? } \\
\text { C. At that point in time } \\
\text { and looking back, what } \\
\text { do you think were the } \\
\text { reasons why you started } \\
\text { masking/ } \\
\text { camouflaging? } \\
\text { D. Do you remember when } \\
\text { and how you started to } \\
\text { realise that you may be } \\
\text { different from others? }\end{array}$ \\
\hline $\begin{array}{l}\text { Part } 2 \text { : Continuity and change } \\
\text { in camouflaging over time }\end{array}$ & $\begin{array}{l}\text { Looking back, has your } \\
\text { camouflaging stayed the } \\
\text { same or changed in any way, } \\
\text { in one or more of the } \\
\text { different areas listed in the } \\
\text { discussion aid }{ }^{\text {an }}\end{array}$ & $\begin{array}{l}\text { A. Do you have some } \\
\text { thoughts on why some } \\
\text { aspects of your } \\
\text { camouflaging may have } \\
\text { changed over time? } \\
\text { Could you share in } \\
\text { more detail? } \\
\text { B. When did you receive } \\
\text { your diagnosis of } \\
\text { autism? Did receiving } \\
\text { the diagnosis change } \\
\text { whether, how much, or } \\
\text { the way you } \\
\text { camouflaged? } \\
\text { C. How would you } \\
\text { describe your } \\
\text { camouflaging/masking } \\
\text { at present? }\end{array}$ \\
\hline
\end{tabular}

${ }^{\mathrm{a}} \mathrm{A}$ discussion aid was presented before the Part 2 opening question listing aspects of camouflaging such as frequency, contexts in which one may camouflage, strategies, motivations, perceived "success" and others. 